\title{
Biomimetic Approach for Liquid Encapsulation with Nanofibrillar Cloaks
}

\author{
Elisa Mele, ${ }^{*}{ }^{\dagger}$ Ilker S. Bayer, $^{\dagger}$ Gabriele Nanni, ${ }^{\dagger}$ José Alejandro Heredia-Guerrero, $^{\dagger}$ Roberta Ruffilli, ${ }^{\ddagger}$ \\ Farouk Ayadi, ${ }^{\dagger}$ Lara Marini, $^{\dagger}$ Roberto Cingolani, ${ }^{\S}$ and Athanassia Athanassiou ${ }^{\dagger}$ \\ ${ }^{\dagger}$ Nanophysics, and ${ }^{\ddagger}$ Nanochemistry, Istituto Italiano di Tecnologia (IIT), Via Morego 30, 16163 Genoa, Italy \\ ${ }^{\S}$ Istituto Italiano di Tecnologia (IIT), Via Morego 30, 16163 Genoa, Italy
}

Supporting Information

ABSTRACT: Technologies that are able to handle microvolumes of liquids, such as microfluidics and liquid marbles, are attractive for applications that include miniaturized biological and chemical reactors, sensors, microactuators, and drug delivery systems. Inspired from natural fibrous envelopes, here, we present an innovative approach for liquid encapsulation and manipulation using electrospun nanofibers. We demonstrated the realization of non-wetting soft solids consisting of a liquid core wrapped in a hydrophobic fibrillar cloak of a fluoroacrylic copolymer and cellulose acetate. By properly controlling the wetting and mechanical properties of the fibers, we created final architectures with tunable mechanical robustness that were stable on a wide range of substrates (from paper to glass) and floated on

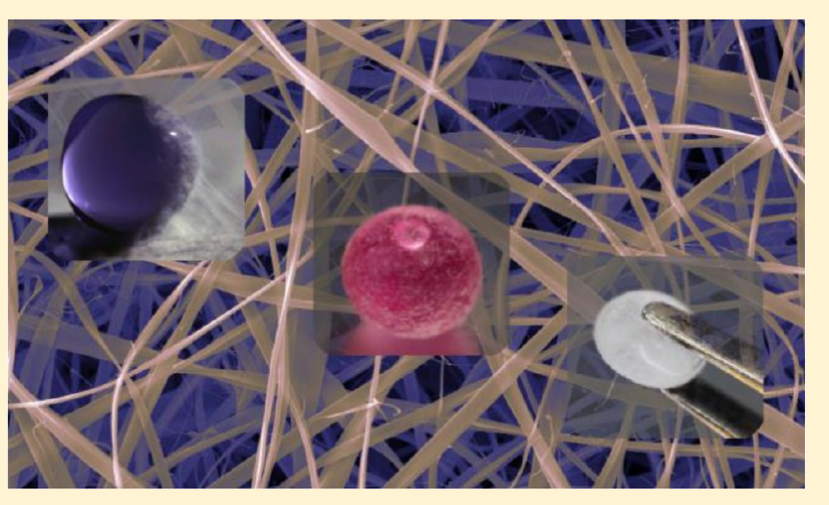
liquid surfaces. Remarkably, the realized fiber-coated drops endured vortex mixing in a continuous oil phase at high stirring speed without bursting or water losses, favoring mixing processes inside the entrapped liquid volume. Moreover, the produced cloak can be easily functionalized by incorporating functional particles, active molecules, or drugs inside the nanofibers.

\section{INTRODUCTION}

Systems for liquid manipulation at the microscale, such as digital microfluidic devices ${ }^{1}$ and liquid marbles, ${ }^{2-6}$ are emerging as miniaturized platforms for chemical and biological processes. They offer advantages in terms of reduced amounts of reagents, shortened reaction velocity, enhanced efficiency, and flexibility. In particular, liquid marbles, namely, drops encapsulated with hydrophobic particles or powders, have been proposed for applications that include gas and liquid sensing, ${ }^{7}$ microreactors, ${ }^{8}$ and water pollution detection. ${ }^{9}$ Because of the great potentialities of these non-wetting droplets, recently, there is a growing interest in improving their mechanical properties that are still unsatisfactory ${ }^{10}$ or developing analogous systems with higher robustness.

The fibrous three-dimensional membranes that animals and vegetables use to encapsulate functional liquids are an alternative and interesting way for bio-inspired fabrication. In fact, capsule-like structures consisting of bundles of collagen fibers exist at the freely movable synovial joints of the human body (such as the hip and knee). ${ }^{11}$ They allow for a large range of movements by reducing the friction between the articular surfaces and delivering nutrients to the cartilages. On the other hand, the fluid-filled volume of each cell in a plant, alga, or fungus is contained in a wall mostly composed of a network of polysaccharide microfibrillars (such as cellulose, mannan, and chitin) that are stabilized in a matrix of proteins and additional polysaccharides. ${ }^{12}$ Cell walls are fundamental structures for cell viability, because they provide mechanical support, shape definition, protection against pathogens, and active nutrient regulation.

Here, inspired by the aforementioned fibrous envelopes, we present the realization of globular structures consisting of a liquid core trapped in a shell of nanofibers and characterized by low friction and mechanical robustness. To this aim, we exploit the impact and rolling of a water drop on a mat of electrospun fibers with engineered wetting properties and morphology. When the chemical composition of the fibers and the impact velocity of the water drops are controlled, the entire liquid volume is wrapped in a nanofibrous cloak. The use of a continuous network of nanofibers instead of particles improves the mechanical resistance of the final system, creating novel architectures mimicking the natural structures.

\section{RESULTS AND DISCUSSION}

The main element of the fiber-coated drops is a mat of hydrophobic polymer blend nanofibers obtained by electrospinning. ${ }^{13}$ The nanofibers consist of a fluoroacrylic copolymer (Capstone ST100) and cellulose acetate (CA). Capstone is an

Received: December 16, 2013

Revised: February 17, 2014

Published: February 24, 2014 

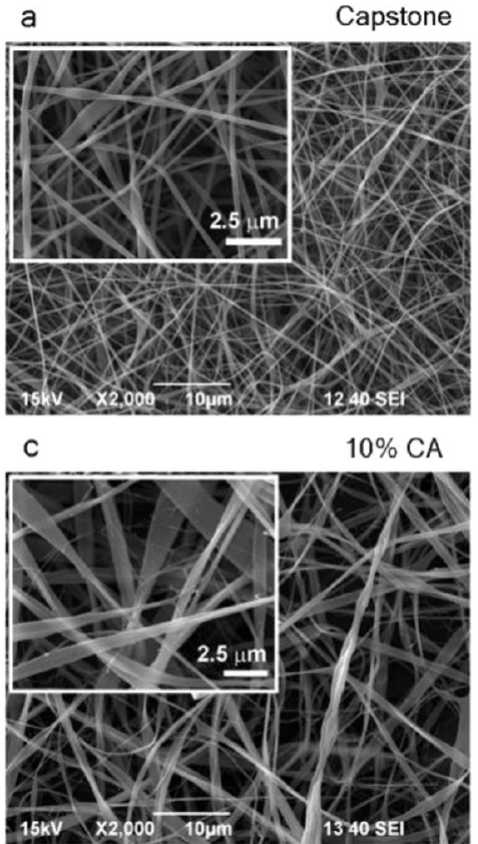

e

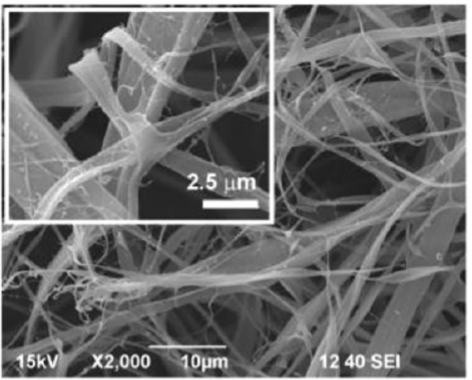

g

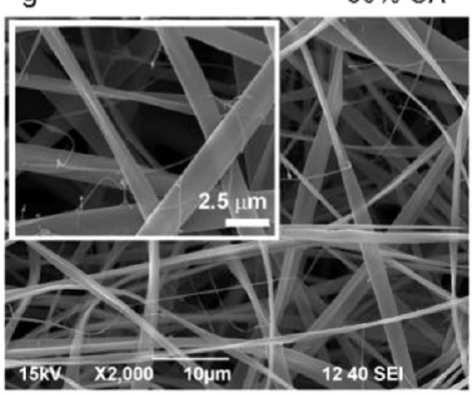

b

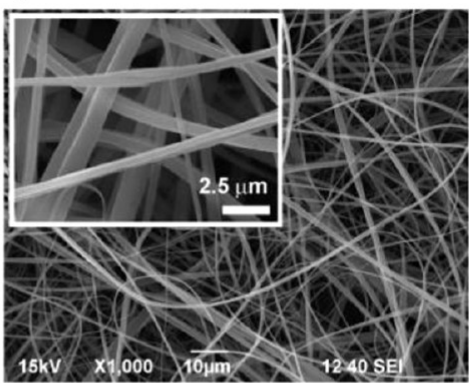

d

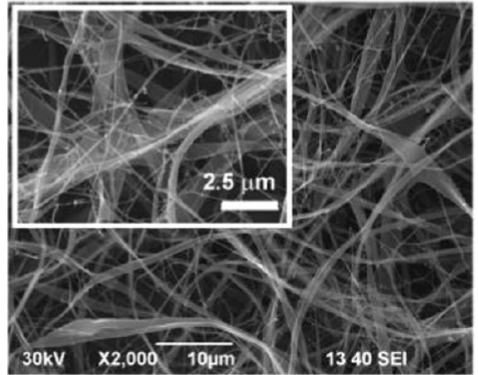

f

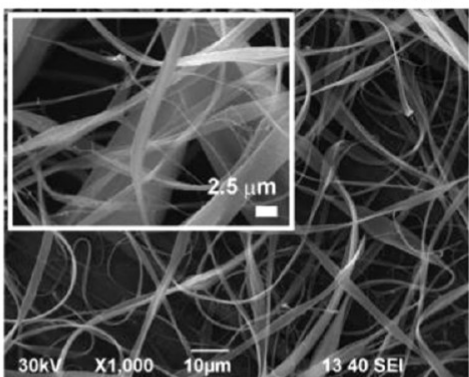

$\mathrm{h}$

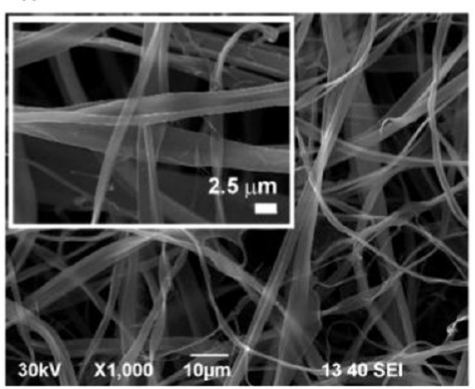

i
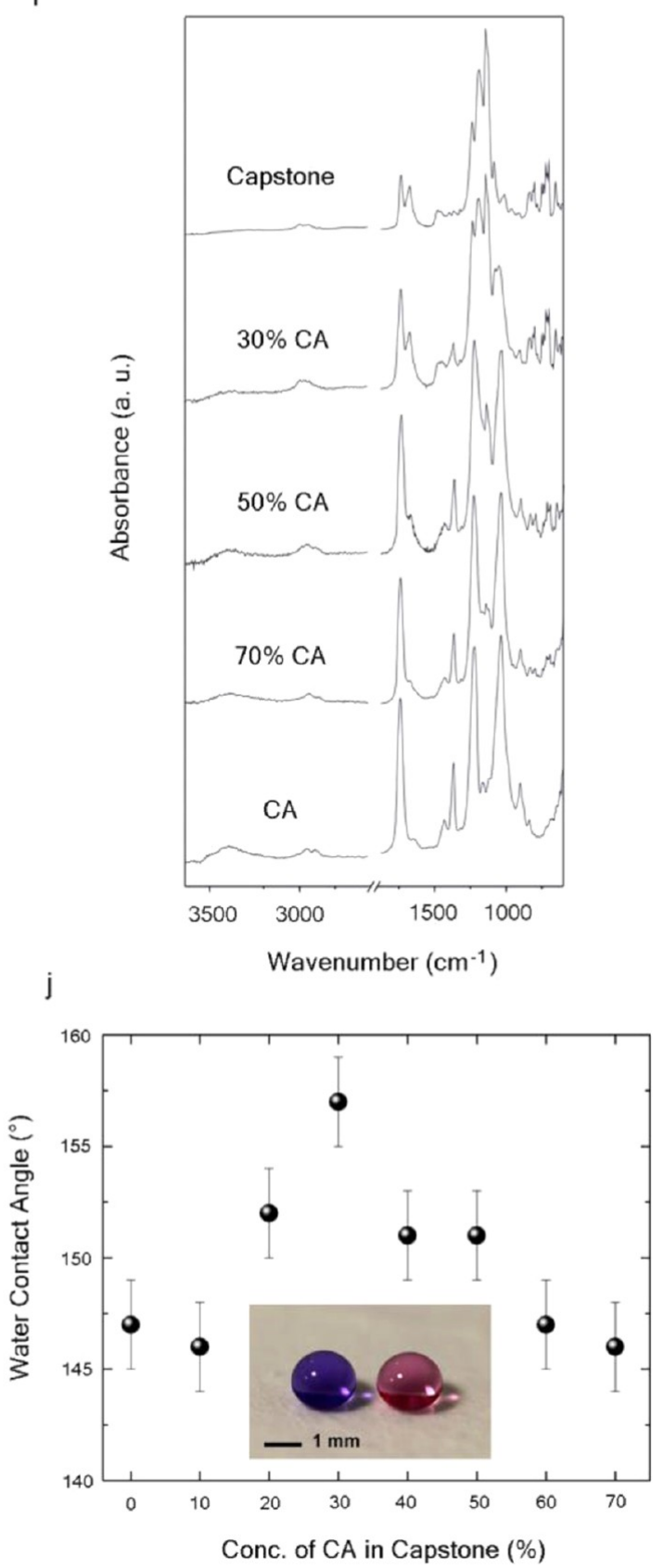

Figure 1. SEM images of electrospun nanofibers consisting of (a) pure Capstone, (b) pure CA, (c) 10\% CA, (d) 20\% CA, (e) 30\% CA, (f) 40\% CA, (g) $50 \% \mathrm{CA}$, and (h) 70\% CA. (Insets) High-magnification SEM micrographs for each Capstone-CA concentration. (i) ATR-FTIR spectra in the $3800-600 \mathrm{~cm}^{-1}$ spectral region of fibrous mats of (from top to bottom) pure Capstone, $30 \% \mathrm{CA}, 50 \% \mathrm{CA}, 70 \% \mathrm{CA}$, and pure CA. (j) Variation of the WCA of the electrospun mats with the CA concentration. (Inset) Photographs of water droplets on the surface of a $30 \%$ CA fibrous substrate showing a WCA higher than $150^{\circ}$.

aqueous fluorochemical dispersion with high water repellency. ${ }^{14}$ To the best of our knowledge, nanofibers based on this polymer are not reported in the literature, probably because the original aqueous formulation of the polymer is not suitable for electrospinning. To electrospin Capstone, we first extracted the fluoroacrylic resin from its water dispersion by trifluoroacetic acid (TFA) and then dissolved it in acetone to obtain a solution at $20 \mathrm{wt} \%$. On the other hand, CA nanofibers are widely reported and used for membranes and filters, scaffolds, drug delivery systems, and textiles, because of their biodegradability and biocompatibility. ${ }^{15}$ Using a combination of solvent inversion and solution blending, Capstone and CA nanofibers were electrospun from acetone as solvent. Figure la shows scanning electron microscopy (SEM) pictures of Capstone nanofibers at different magnifications. The fibers exhibit a smooth morphology without beaded structures and have a diameter $(d)$ of $280 \pm 100 \mathrm{~nm}$. Nanofibers of pure CA were electrospun from a solution at $15 \mathrm{wt} \%$ in acetone, producing ribbon-like fibers with $d \cong 550 \pm 130 \mathrm{~nm}$ (Figure $1 \mathrm{~b}$ ). Micrometer-sized fibers are also present, because of incomplete solvent evaporation. Combining the two polymers at different concentrations (from 10 to 70 wt \% of CA in Capstone, in steps of $10 \%$ ), we were able to form unique fibrous mats with complex topographies. Panels $\mathrm{c}-\mathrm{h}$ of Figure 1 show that 
Impact

a

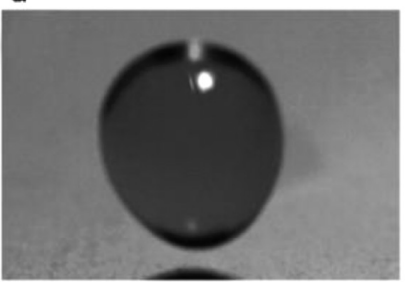

b

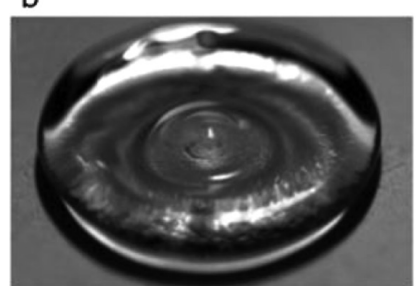

$8 \mathrm{~ms}$

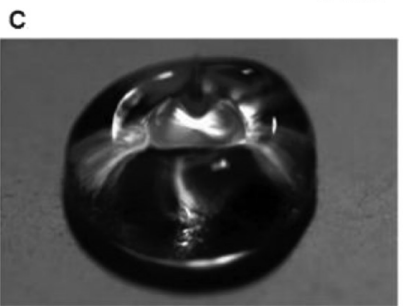

$16 \mathrm{~ms}$ d

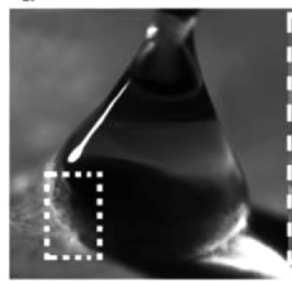

e

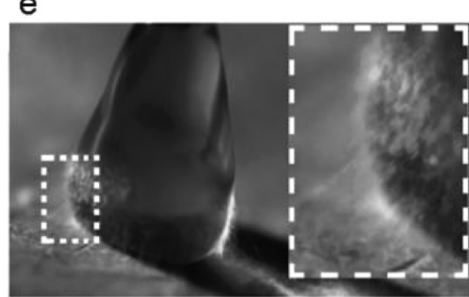

f

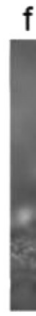

$24 \mathrm{~ms}$

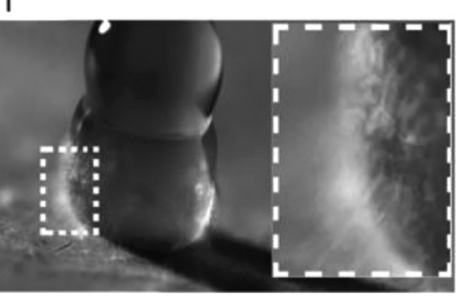

$28 \mathrm{~ms}$ g

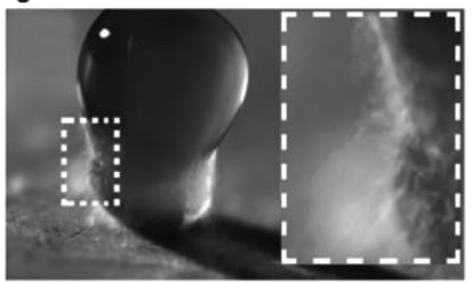

$32 \mathrm{~ms}$

h
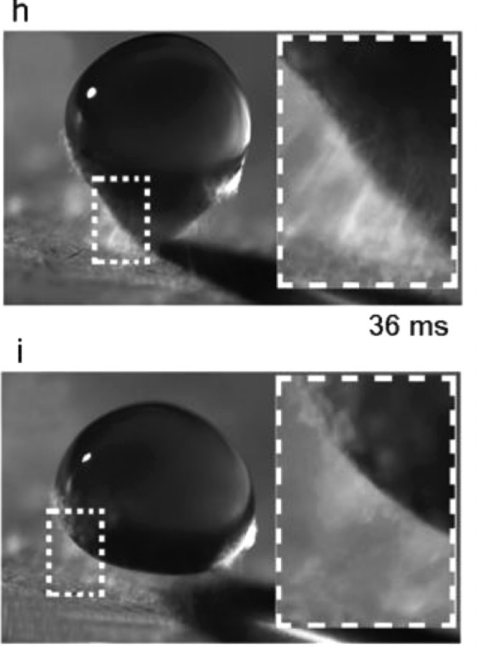

$40 \mathrm{~ms}$
Rolling

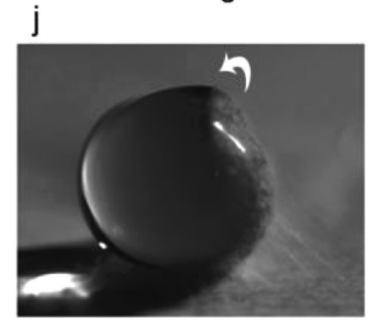

k

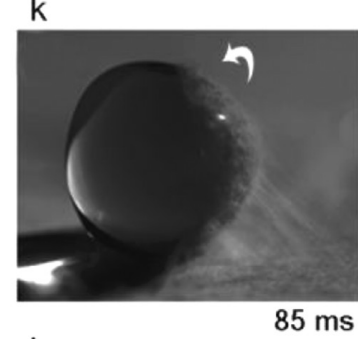

I

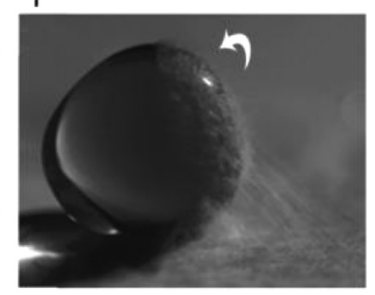

$90 \mathrm{~ms}$

Figure 2. Sequential optical images recorded by a high-speed camera at $4000 \mathrm{fps}$ of $30 \mu \mathrm{L}$ of water drop: (a-i) impacting with a velocity of $0.8 \mathrm{~m} / \mathrm{s}$ on the surface of a pure Capstone fibrous mat and ( $j-1)$ rolling on the surface after impact. (Insets in panels $d-i$ ) High-magnification pictures showing the peeling of the fibers from the substrate and how they are collected on the water drop surface.

increasing the amount of CA fibers with different shapes and cross-sectional diameters coexist in the same network. This is particularly evident in mats containing 20 and 30\% CA (panels $\mathrm{d}$ and e of Figure 1, respectively), where ultrafine nanofibers of diameter in the range of 50-300 $\mathrm{nm}$ are superimposed and entangled around bigger ribbon-like fibers (inset in Figure 1e), generating structures with multi-scale features.

Previous studies have described the production of similar hierarchical fibrous networks by electrohydrodynamics processes, such as multiple-jet electrospinning based on the use of separate spinnerets ${ }^{16}$ or compartments ${ }^{17}$ or electronetting that exploits phase-separation-induced splitting of charged droplets in high electric fields. ${ }^{18}$ In our case, complex multi-scale architectures could be attributed to the presence of two separate phases or concentration gradients in the starting solution, as a consequence of the incomplete miscibility of Capstone ST100 and CA. To better investigate this point, we characterized the nanofibers by attenuated total reflectance coupled to a Fourier transform infrared (ATR-FTIR) spectrometer and thermogravimetric analysis (TGA). As shown in Figure 1i, pure Capstone ST100 fibers exhibit bands associated to $-\mathrm{CF}_{2}-$ groups (asymmetric and symmetric $\mathrm{CF}_{2}$ stretching at 1234 and $1142 \mathrm{~cm}^{-1}$, respectively), ester functional groups $\left(\mathrm{C}=\mathrm{O}\right.$ stretching at $1732 \mathrm{~cm}^{-1}$ and asymmetric $\mathrm{C}-\mathrm{O}-\mathrm{C}$ stretching at $\left.1184 \mathrm{~cm}^{-1}\right), \mathrm{C}=\mathrm{C}$ groups $\left(\mathrm{C}=\mathrm{C}-\mathrm{H}\right.$ stretching at $2997 \mathrm{~cm}^{-1}$ and $\mathrm{C}=\mathrm{C}$ stretching at $1670 \mathrm{~cm}^{-1}$ ), and hydroxyl groups (-OH stretching at 3464 $\left.\mathrm{cm}^{-1}\right){ }^{19,20}$ On the other hand, characteristic peaks of CA are evident in pure CA fibers: ${ }^{21}-\mathrm{OH}$ stretching at $3497 \mathrm{~cm}^{-1}$, asymmetric and symmetric $\mathrm{CH}_{2}$ stretching at 2945 and 2885 $\mathrm{cm}^{-1}$ respectively, $\mathrm{C}=\mathrm{O}$ stretching at $1740 \mathrm{~cm}^{-1}$, acetyl group $\mathrm{C}-\mathrm{O}$ stretching at $1221 \mathrm{~cm}^{-1}$, asymmetric stretching of the $\mathrm{C}-$ $\mathrm{O}-\mathrm{C}$ bridge at $1163 \mathrm{~cm}^{-1}$, and stretching of pyranose ring $\mathrm{C}-$ $\mathrm{O}-\mathrm{C}$ at $1036 \mathrm{~cm}^{-1}$. In the spectra of the composite fibers (30, 50 , and $70 \% \mathrm{CA}$ ), we see neither significant evidence of shifts in the position of the characteristic peaks of Capstone and CA nor the formation/disappearance of bands. This indicates that the two polymers are solely blended in the solution and only weak interactions may be established between them. Moreover, the TGA thermograms of the composite fibers (see Figure S1 of the Supporting Information) reveal that their degradation behavior is a combination of the thermal profiles of Capstone and CA. For Capstone, in fact, three weight losses are seen at $100{ }^{\circ} \mathrm{C}$ because of the evaporation of the absorbed water or remaining solvent, at $215^{\circ} \mathrm{C}$ associated with the elimination of the acrylic groups, ${ }^{22}$ and in the range of $300-420{ }^{\circ} \mathrm{C}$ related to fluorinated groups. ${ }^{23} \mathrm{~A}$ similar three-step thermal degradation is observed in the composite fibers, where the last weight loss is also due to the contribution of CA that has a single transition temperature at $361{ }^{\circ} \mathrm{C} .{ }^{24}$ We therefore conclude that Capstone and CA form a physical polymeric blend that results in the formation of multi-scale electrospun fibers.

The complex topography and the resulting wetting properties of these electrospun mats play an essential role in the formation of nanofibrous cloaks around liquid drops. As presented in Figure 1j, pure Capstone nanofiber mats exhibit a hydrophobic character with a water contact angle (WCA) of about $147^{\circ}$; instead, pure CA fibers have an initial WCA of $134^{\circ}$, decreasing with time to $0^{\circ}$ (full absorption). We observed that an increase of CA in the Capstone matrix, from 10 to $30 \%$, 

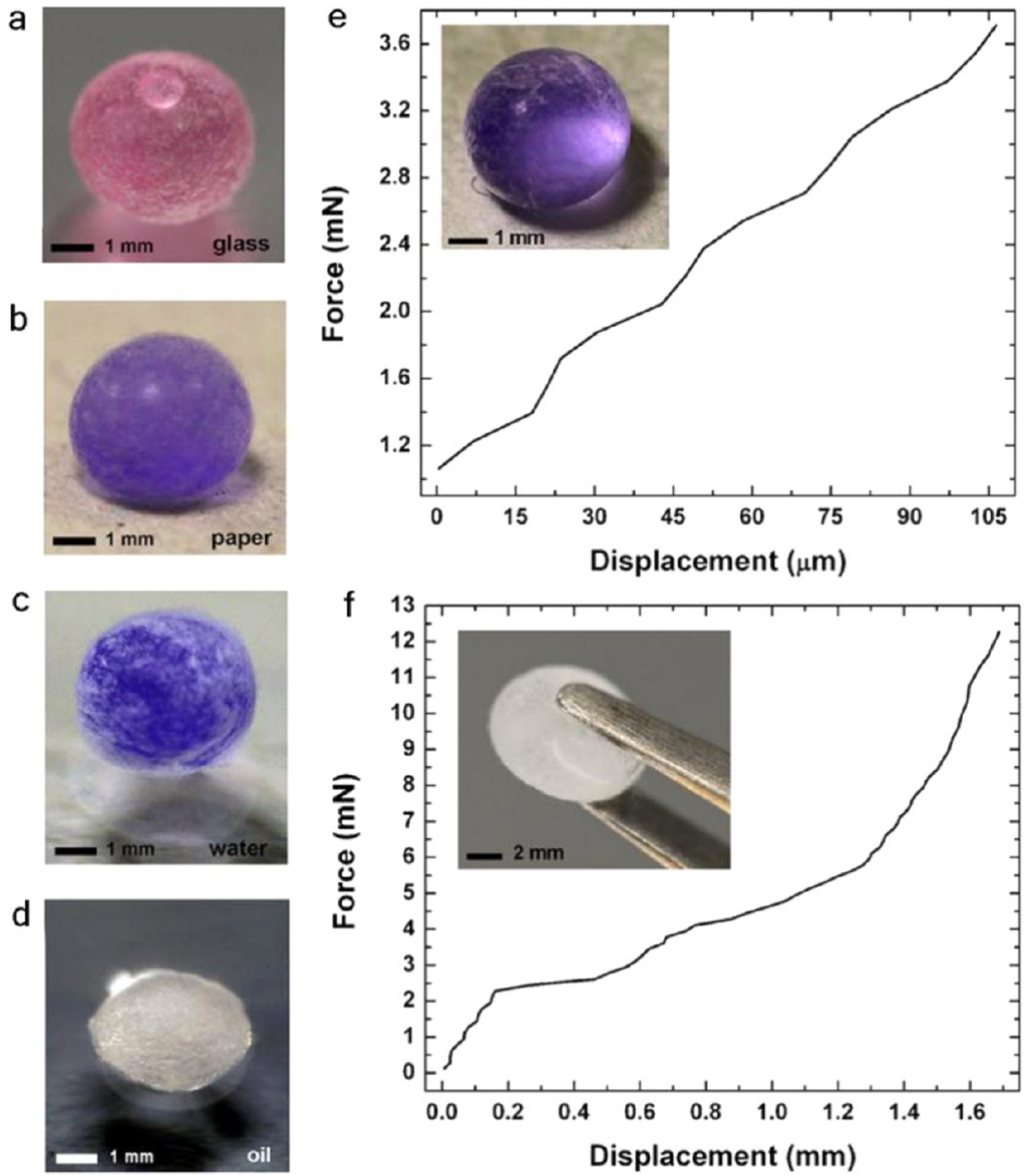

Figure 3. Photographs of fiber-coated drops on (a) glass, (b) paper, (c) floating on water, and (d) floating on silicone oil. Compression forcedisplacement curves of water drops wrapped with (e) 60\% CA fibers and (f) $70 \%$ CA fibers. (Insets in panels e and f) Photographs of the fibercoated drops before and after compression, respectively. To show clearly the mechanical deformation, tweezers were used to apply the compressive load.

raises the WCA up to $157^{\circ}$ for fibers with $30 \mathrm{wt} \% \mathrm{CA}$. For CA concentrations from 40 to $70 \%$, the WCA decreases to $146^{\circ}$. On the basis of these observations, we divided the produced fiber mats into two classes according to their wetting properties: (a) class A includes fibers of pure Capstone or containing a concentration CA of $<20$ or $>50 \%$, and they are hydrophobic with WCA $<150^{\circ}$, droplet sliding angle $\cong 45^{\circ}$, and contact angle hysteresis $(\mathrm{CAH}) \sim 85-90^{\circ}$; (b) class $\mathrm{B}$ includes fibers with $20 \leq \mathrm{CA} \leq 50 \%$, and they are superhydrophobic with WCA $>150^{\circ}$, droplet sliding angle $\sim 25-30^{\circ}$, and $\mathrm{CAH} \sim 40-65^{\circ}$. From these two classes, we observed that only fibers belonging to class A are suitable for wrapping water drops.

In Figure 2, the dynamics of the formation of fiber-coated drops is presented, recorded by a high-speed camera at 4000 fps. A $30 \mu \mathrm{L}$ water drop was released from a height of about 2.5 $\mathrm{cm}$ on the surface of a Capstone fibrous substrate. After the drop impacts with a velocity of about $0.7 \mathrm{~m} / \mathrm{s}$, it spreads on the surface, forming a circular lamella (panels a-c of Figure 2). After the maximum spreading diameter is reached (Figure $2 b$ ), the drop continues changing shape moving upward (panels $\mathrm{d}-$ $\mathrm{h}$ of Figure 2), eventually detaching from the fibrous mat (Figure 2i). The considerable deformation that the drop undergoes during spreading and retracting is due to the excess of kinetic energy with respect to the surface energy. The ratio between both quantities is the Weber number $(W e)$, defined as
$W e=\rho v^{2} d / \sigma$, where $\rho$ is the density of the liquid, $v$ is the impact velocity, $d$ is the drop diameter, and $\sigma$ is the surface tension of the liquid drop. ${ }^{25}$ For the analyzed drops, we calculated high $W e$ values (around 20). In the insets in panels $\mathrm{d}-\mathrm{i}$ of Figure 2, it is clearly visible that, during impact and rebound, the water drop extirpates nanofibers from the mat and collects them on its own surface. We believe that the liquid drop penetrates inside the voids of the fibrous web because of the high $\mathrm{CAH}^{26}$ and has enough energy to take away a thin layer of fibers. This could be due to the fact that Capstone fibers are not strongly entangled between them, as previously described, and superficial layers can be easily detached from the electrospun network. In this initial phase (up to $40 \mathrm{~ms}$ after impact), the drop is only partially covered with the nanofibers, because the contact area between the liquid and the fibers was limited to the spreading region. After the drop bounced off from the surface, it falls again on the surface with much lower kinetic energy; this time. This causes the drop to roll on the fibrous mat with the collection of bundles of fibers (panels $j-1$ of Figure 2). When the drop stops moving, further drop rolling is forced by gently shaking the mat-coated substrate, allowing the additional attachment of fibers and the eventual coverage of the entire water volume. At the end of the process (less than 5 s), the water drop is completely wrapped in a shell of nanofibers. 
We point out that the fibers belonging to class $A$ can be used to encapsulate liquids, contrary to those of class B. In the latter case, in fact, when the drop impacts on the superhydrophobic mat, it maintains its spherical shape without collecting fibers. Consequently, no drop coverage is observed, probably because the complex hierarchical topography and the relative wetting properties of the mat limit the water penetration inside the voids between the fibers. Furthermore, when the concentration of CA exceeds $70 \%$, the impacting drop spreads without beading back up on the fibrous surface and without being encapsulated, because of the hygroscopic character of the CA fibers. In panels a-e of Figure 3 and in the inset in Figure 3f, photographs of such fiber-coated drops on different surfaces are shown. We found that they are stable on a wide range of substrates, including glass (Figure 3a), paper (Figure 3b), aluminum (inset in Figure 3f), other metals, and plastics, and they can also float on water (Figure $3 \mathrm{c}$ ) and silicone oil (Figure 3d). Floating fiber-coated drops exhibited long lifetimes (2-3 h) without collapse or bursting when they were deposited on the water (oil) interface. Only a gradual shrinking was observed because of the evaporation of the liquid inside the fibrous cloak.

Some of the developed fiber-coated drops exhibit remarkable mechanical robustness depending upon the fibers used for the encapsulation. Here, we discuss the compression test of water drops with a volume of $15 \mu \mathrm{L}$ (radius of $1.5 \mathrm{~mm}$ ) wrapped with fibers belonging to class $\mathrm{A}$, whose mechanical behavior was investigated by a dynamic mechanical analyzer. We observed that drops coated with fibers of pure Capstone or 10\% CA had low mechanical resistance and endured a maximum compression force $\left(F_{\max }\right)$ of $0.1 \mathrm{mN}$. When the specific fiber-coated drops were compressed using $F_{\text {max }}$ they significantly deformed (from spherical to disk shape), increasing the contact area with the parallel squeezing plates. Fractures in the fibrous shells originated with consequent leakage of the encapsulated water. Instead, when fibrous mats with 60 or $70 \%$ CA were used, robust fiber-coated drops were produced, resisting up to $F_{\max }$ of 3.7 and $12.3 \mathrm{mN}$, respectively. In panels e and $\mathrm{f}$ of Figure 3, compression force-displacement curves recorded for drops coated with 60 and $70 \%$ CA fibers are shown. A linear elastic behavior is observed for drops wrapped in 60\% CA fibers; in the case of drops coated with $70 \%$ CA fibers, they initially deform elastically and then plastically, undergoing a compression of around $50 \%$ of their initial diameter before bursting (inset in Figure 3f). The enhanced robustness of the drops is very likely due to a combination of the larger fiber shell thickness and enhanced mechanical properties. In fact, with increasing the CA concentration, the thickness of the coverage around the liquid drop increases from $5 \mu \mathrm{m}$ for pure Capstone to $15 \mu \mathrm{m}$ for $70 \%$ CA fibers. In addition, tensile tests carried out on the non-woven mats revealed that Capstone fibers are more brittle and fragile than fibers containing 70\% CA. The Capstone network is indeed less extensible than the CA-based network, and then it abruptly breaks, showing a sharp and smooth fracture (see Figure S2a of the Supporting Information). The elongation before breaking of the Capstone fibrous mat is about one-half of that measured for $70 \% \mathrm{CA}$ fibers. The latter is instead more intertwined with each other, and the mat is able to undergo larger deformations upon applied external stress (see Figure S2b of the Supporting Information). Consequently, because drops covered with $70 \%$ CA fibers are characterized by a thick and less fragile fibrous shell, they can reach high bursting forces. The comparison between our novel fiber-coated drops and conventional liquid marbles based on micro- and nanopowders reveals that the structures presented here are able to endure compressive loads up to 10 times higher. In fact, works on liquid marbles have reported typical breaking forces in the range of $1-2 \mathrm{mN},{ }^{27}$ with a record of $6 \mathrm{mN}$ for specific designed microparticles. ${ }^{28}$

Moreover, we analyzed the robustness of the realized fibercoated drops in a continuous oily phase. We observed that the structures remained stable even when they were forced to sink inside silicon oil and no bursting was noted after their contact with the bottom of the glass container (Figure 4a). In oil, water evaporation is strongly reduced and the shape of the spheres is preserved for months. To demonstrate the mechanical stability of these liquid spheres in oil, we exposed them to vortex mixing at a stirring speed of $3000 \mathrm{rpm}$. In panels b-e of Figure 4,

\section{a}
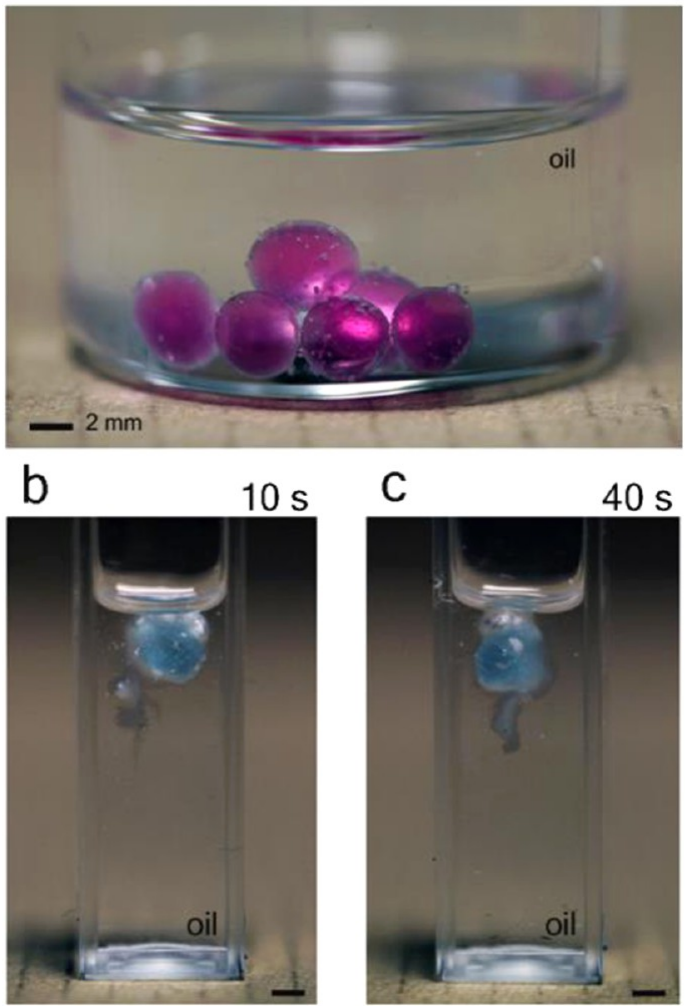

C
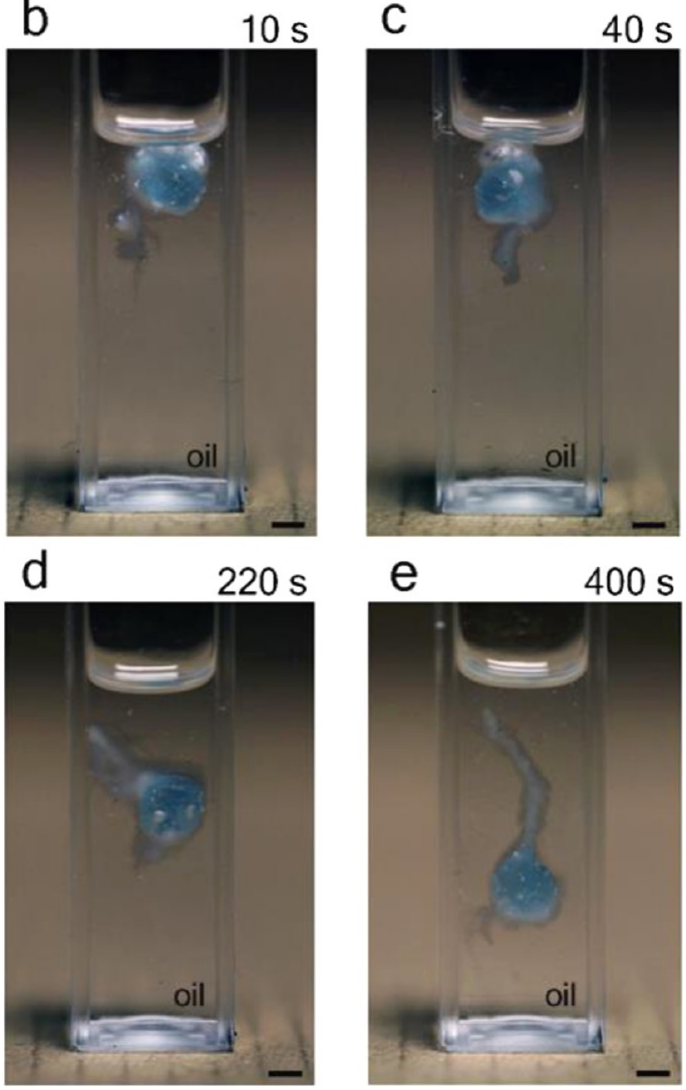

e $400 \mathrm{~s}$

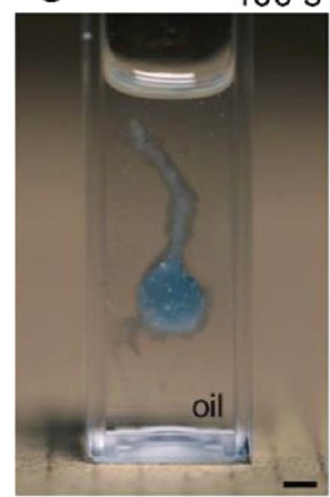

Figure 4. Photographs of (a) water droplets coated with $60 \%$ CA nanofibers completely immersed in silicone oil and $(b-e)$ one single sphere after shaking in oil for $10,40,220$, and $400 \mathrm{~s}$, respectively. Different dyes were used to color the water and make the structures clearly visible. Scale bar $=0.2 \mathrm{~cm}$. 
photographs of one single sphere after different stirring times are shown. Remarkably, the aqueous volume remains encapsulated within the fibrous shell even after $400 \mathrm{~s}$ of vortex mixing and is not defragmented in many small droplets, as opposed to a non-coated water drop that is instead split up after 5 s. Certainly, the nanofibrous cloak is weakened by the stirring process and starts to unravel, forming the long thread clearly visible in Figure 4e. Nevertheless, the nanofibers still work synergistically, like a continuous net, to maintain the structural integrity of the wrapped water drop.

\section{CONCLUSION}

We conclude that the encapsulation of liquid volumes with electrospun nanofiber is an effective way to produce nonwetting soft solids with superior robustness than conventional liquid marbles. This approach offers a high degree of flexibility because the properties of the nanofibrillar cloak can be modified by incorporating functional particles, active molecules, and drugs. ${ }^{29}$ Furthermore, the stability of the fiber-coated drops on different types of substrates and the possibility to carry out mixing processes inside the liquid volumes make them suitable for the realization of miniaturized bioreactors, sensors, microactuators, and drug delivery systems.

\section{MATERIALS AND METHODS}

Materials. Capstone ST100 was purchased from Dupont; it is an aqueous fluorochemical dispersion, containing $74-81 \%$ water, $19-$ $26 \%$ of a fluorinated acrylic copolymer, and $0.003-0.01 \%$ tetramethyl succinonitrile. CA (acetyl content of $39.8 \% ; M_{\mathrm{w}}=30 \mathrm{kDa}$ ), acetone, TFA, and silicone oil $(50 \mathrm{cSt})$ were obtained from Sigma-Aldrich and used as received.

Electrospinning Procedure. Solutions of Capstone in acetone were obtained following a two-step process. First, the polymer was extracted from the original aqueous dispersion by mixing equal volumes of Capstone and TFA. The precipitated polymer was washed in water to eliminate the acid residues and then dissolved in acetone at a concentration of $20 \mathrm{wt} \%$. Solutions of CA in acetone were obtained at $15 \mathrm{wt} \%$. Acetone-based solutions with different concentrations of $\mathrm{CA}$ in Capstone were also prepared, from 10 to $70 \mathrm{wt} \%$ with increments of $10 \%$. A plastic syringe with a stainless-steel 23-gauge needle was filled with the obtained solutions and connected to a syringe pump (NE-1000, New Era Pump Systems, Inc.) working at a flow rate of $3 \mathrm{~mL} \mathrm{~h}^{-1}$. The solutions were electrospun at a voltage of $20 \mathrm{kV}$, controlled using a high-voltage power supply (EH40R2.5, Glassman High Voltage, Inc.). The distance between the needle and the aluminum collector was fixed at $15 \mathrm{~cm}$.

Characterizations. The morphology of the produced nanofibers was characterized by SEM, using a JEOL JSM-6490LA microscope working in high-vacuum mode, with an acceleration voltage of $15 \mathrm{kV}$. Infrared spectra of the fibrous samples were obtained with an attenuated total reflectance (ATR) accessory (MIRacle ATR, PIKE Technologies) coupled to a Fourier transform infrared (FTIR) spectrometer (Equinox $70 \mathrm{FT}$-IR, Bruker). All spectra were recorded in the range from 4000 to $600 \mathrm{~cm}^{-1}$, with $4 \mathrm{~cm}^{-1}$ resolution (accumulating 128 scans). In a typical measurement, the sample was gently collocated on the spot of ATR accessory and slowly pressed. The thermal degradation behavior of the nanofibers was investigated by means of TGA using a TGA Q500 from TA Instruments. Measurements were performed under an inert $\mathrm{N}_{2}$ atmosphere with a flow rate of $50 \mathrm{~mL} / \mathrm{min}$ in a temperature range from 30 to $600{ }^{\circ} \mathrm{C}$ at heating rate of $10^{\circ} \mathrm{C} / \mathrm{min}$. The weight loss [thermogravimetry (TG) curve] was recorded as a function of time/temperature.

To measure the hydrophobicity of the electrospun nanofibers, thick mats were spun on microscope glass slides. Contact angle measurements were carried out using a standard contact angle goniometer equipped with a charge-coupled device (CCD) camera and image processing software. Up to 10 contact angle measurements were carried out on every sample at random locations, and their average values are reported. Sliding angle measurements were made by tilting the substrates slowly until the droplets rolled off the substrates. Contact angle hysteresis measurements were made by liquid injection (for advancing the contact angle) and liquid suction (for receding the contact angle) techniques. A sessile droplet $(\sim 15 \mu \mathrm{L})$ attached to the tip of the needle was gently lowered to the surfaces to form an equilibrium contact angle. After this, water was injected into the droplet very slowly (without increasing the droplet size significantly) to monitor if the contact line moves (advances) over the surfaces. In the case of advancing contact line, an average angle was recorded as an advancing contact angle. Similarly, for the receding contact angle, water was slowly sucked away from the droplet and the state of the contact line was monitored. In the case of the moving contact line, an average measurement was recorded as the receding contact angle.

Videos at high frame rate are obtained by a high-speed camera IDT MotionXtra NX4S2, working at a maximum frame rate of $9090 \mathrm{~Hz}$.

The mechanical properties of the fiber-coated drops and fibrous networks were investigated by a dynamic mechanical analyzer (Q800, TA Instruments) in compression and tension mode, respectively. The compression tests were performed by placing the fiber-coated drops between a pair of clean parallel plates, one fixed and one movable. Because water evaporation may significantly affect their mechanical strength, freshly formed fiber-coated drops were analyzed. After a compressive preloading of 0.1 and $1 \mathrm{mN}$ was applied in normal direction for $70 \%$ and $60 \%$ CA fibers respectively, a ramp force of 10 $\mathrm{mN} / \mathrm{min}$ was applied until the rapture occurred. Moreover, tension tests were carried out on the fibrous mats with a ramp displacement of $250 \mu \mathrm{m} / \mathrm{min}$.

\section{ASSOCIATED CONTENT}

\section{Supporting Information}

TGA thermograms of the composite fibers (Figure S1) and photographs of the fracture that occurred in fibrous mats of (a) pure Capstone and (b) 70\% CA after the application of a tensile stress (Figure S2). This material is available free of charge via the Internet at http://pubs.acs.org.

\section{AUTHOR INFORMATION}

\section{Corresponding Author}

*E-mail: elisa.mele@iit.it.

\section{Notes}

The authors declare no competing financial interest.

\section{REFERENCES}

(1) Choi, K.; Ng, A. H. C.; Fobel, R.; Wheeler, A. R. Digital microfluidics. Annu. Rev. Anal. Chem. 2012, 5, 413-440.

(2) Aussillous, P.; Quéré, D. Liquid marbles. Nature 2001, 411, 924927.

(3) McHale, G.; Newton, M. I. Liquid marbles: Principles and applications. Soft Matter 2011, 7, 5473-5481.

(4) Bormashenko, E.; Bormashenko, Y.; Musin, A.; Barkay, Z. On the mechanism of floating and sliding of liquid marbles. ChemPhysChem 2009, 10, 654-656.

(5) Mengmeng, C.; Emrick, T.; Russell, T. P. Stabilizing liquid drops in nonequilibrium shapes by the interfacial jamming of nanoparticles. Science 2013, 342, 460-463.

(6) Mertaniemi, H.; Laukkanen, A.; Teirfolk, J.-E.; Ikkala, O.; Ras, R. H. A. Functionalized porous microparticles of nanofibrillated cellulose for biomimetic hierarchically structured superhydrophobic surfaces. RSC Adv. 2012, 2, 2882-2886.

(7) Tian, J.; Arbatan, T.; Li, X.; Shen, W. Liquid marble for gas sensing. Chem. Commun. 2010, 46, 4734-4736.

(8) Arbatan, T.; Lizi, L.; Tian, J.; Shen, W. Liquid marbles as microbioreactors for rapid blood typing. Adv. Healthcare Mater. 2012, 1, 8083. 
(9) Bormashenko, E.; Musin, A. Revealing of water surface pollution with liquid marbles. Appl. Surf. Sci. 2009, 255, 6429-6431.

(10) Chin, J. M.; Reithofer, M. R.; Tan, T. T. Y.; Menon, A. G.; Chen, E. Y.; Chow, C. A.; Hora, A. T. S.; Xu, J. Supergluing MOF liquid marbles. Chem. Commun. 2013, 49, 493-495.

(11) Ralphs, J. R.; Benjamin, M. The joint capsule: Structure, composition, ageing and disease. J. Anat. 1994, 184, 503-509.

(12) Somerville, C.; Bauer, S.; Brininstool, G.; Facette, M.; Hamann, T.; Milne, J.; Osborne, E.; Paredez, A.; Persson, S.; Raab, T.; Vorwerk, S.; Youngs, H. Toward a systems approach to understanding plant cell walls. Science 2004, 306, 2206-2211.

(13) Nuraje, N.; Khan, W. S.; Lei, Y.; Ceylan, M.; Asmatulu, R. Superhydrophobic electrospun nanofibers. J. Mater. Chem. A 2013, 1, 1929-1946.

(14) Bayer, I. S.; Steele, A.; Loth, E. Superhydrophobic and electroconductive carbon nanotube-fluorinated acrylic copolymer nanocomposites from emulsions. Chem. Eng. J. 2013, 221, 522-530.

(15) Konwarh, R.; Karak, N.; Misra, M. Electrospun cellulose acetate nanofibers: The present status and gamut of biotechnological applications. Biotechnol. Adv. 2013, 31, 421-437.

(16) Soliman, S.; Paglian, S.; Rinaldi, A.; Forte, G.; Fiaccavento, R; Pagliari, F.; Franzese, O.; Minieri, M.; Di Nardo, P.; Licoccia, S.; Traversa, E. Multiscale three-dimensional scaffolds for soft tissue engineering via multimodal electrospinning. Acta Biomater. 2010, 6, 1227-1237.

(17) Grey, C. P.; Newton, S. T.; Bowlin, G. L.; Haas, T. W.; Simpson, D. G. Gradient fiber electrospinning of layered scaffolds using controlled transitions in fiber diameter. Biomaterials 2013, 34, 4993-5006.

(18) Wang, X.; Ding, B.; Sun, G.; Wang, M.; Yu, J. Electro-spinning/ netting: A strategy for the fabrication of three-dimensional polymer nano-fiber/nets. Prog. Mater. Sci. 2013, 58, 1173-1243.

(19) Liang, C. Y.; Krimm, S. Infrared spectra of high polymers. III. Polytetrafluoroethylene and polychlorotrifluoroethylene. J. Chem. Phys. 1956, 25, 563-571.

(20) Bellamy, L. J. The Infrared Spectra of Complex Molecules; Chapman and Hall: New York, 1975.

(21) Gulec, H. A.; Topacli, A.; Topacli, C.; Albayrak, N.; Mutlu, M. Modification of cellulose acetate membrane via low-pressure plasma polymerization for sugar separation applications: Part I. Membrane development and characterization. J. Membr. Sci. 2010, 350, 310-321.

(22) Xiong, C.; Yao, C. Preparation and application of acrylic acid grafted polytetrafluoroethylene fiber as a weak acid cation exchanger for adsorption of $\operatorname{Er}(\mathrm{III})$. J. Hazar. Mater. 2009, 170, 1125-1132.

(23) Smith, D. W.; Babb, D. A. Perfluorocyclobutane aromatic polyethers. Synthesis and characterization of new siloxane-containing fluoropolymers. Macromolecules 1996, 29, 852-860.

(24) Lucena, M. C. C.; de Alencar, A. E. V.; Mazzeto, S. E.; Soares, S. A. The effect of additives on the thermal degradation of cellulose acetate. Polym. Degrad. Stab. 2003, 80, 149-155.

(25) Leng, L. J. Splash formation by spherical drops. J. Fluid Mech. 2001, 427, 73-105.

(26) Kannan, R,; Vaikuntanathan, V.; Sivakumar, D. Dynamic contact angle beating from drops impacting onto solid surfaces exhibiting anisotropic wetting. Colloids Surf., A 2011, 386, 36-44.

(27) Lai, Y.; Tang, Y.; Huang, J.; Wang, H.; Li, H.; Gong, D.; Ji, X.; Gong, J.; Lin, C.; Sun, L.; Chen, Z. Multi-functional hybrid protonated titanate nanobelts with tunable wettability. Soft Matter 2011, 7, 63136319.

(28) Zang, D.; Chen, Z.; Zhang, Y.; Lin, K.; Geng, X.; Binks, B. P. Effect of particle hydrophobicity on the properties of liquid water marbles. Soft Matter 2013, 9, 5067-5073.

(29) Bayer, I. S.; Fragouli, D.; Attanasio, A.; Sorce, B.; Bertoni, G.; Brescia, R.; Di Corato, R.; Pellegrino, T.; Kalyva, M.; Sabella, S.; Pompa, P. P.; Cingolani, R.; Athanassiou, A. Water-repellent cellulose fiber networks with multifunctional properties. ACS Appl. Mater. Interfaces 2011, 3, 4024-4031. 